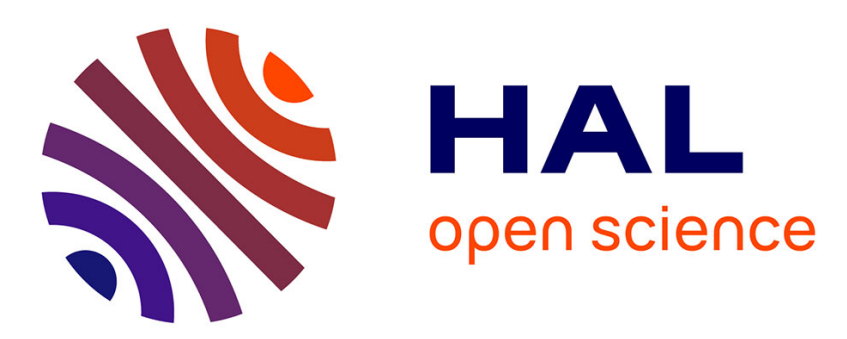

\title{
Throughput optimization for micro-factories subject to task and machine failures
}

\author{
Anne Benoit, Alexandru Dobrila, Laurent Philippe, Jean-Marc Nicod
}

\section{To cite this version:}

Anne Benoit, Alexandru Dobrila, Laurent Philippe, Jean-Marc Nicod. Throughput optimization for micro-factories subject to task and machine failures. APDCM'10, 12th Workshop on Advances on Parallel and Distributed Processing Symposium, 2010, United States. pp.11-18. hal-00563628

\section{HAL Id: hal-00563628 \\ https://hal.science/hal-00563628}

Submitted on 7 Feb 2011

HAL is a multi-disciplinary open access archive for the deposit and dissemination of scientific research documents, whether they are published or not. The documents may come from teaching and research institutions in France or abroad, or from public or private research centers.
L'archive ouverte pluridisciplinaire HAL, est destinée au dépôt et à la diffusion de documents scientifiques de niveau recherche, publiés ou non, émanant des établissements d'enseignement et de recherche français ou étrangers, des laboratoires publics ou privés. 


\section{Throughput optimization for micro-factories subject to task and machine failures}

\author{
Anne Benoit \\ ENS Lyon, Université de Lyon, France \\ LIP laboratory (ENS, CNRS, INRIA, UCBL) \\ Anne.Benoit@ens-lyon.fr
}

\author{
Alexandru Dobrila, Jean-Marc Nicod and Laurent Philippe \\ Laboratoire d'Informatique de Franche-Comté, \\ Université de Franche-Comté, France \\ adobrila,jmnicod,lphilippe@lifc.univ-fcomte.fr
}

\begin{abstract}
In this paper, we study the problem of optimizing the throughput for micro-factories subject to failures. The challenge consists in mapping several tasks of different types onto a set of machines. The originality of our approach is the failure model for such applications in which not only the machines are subject to failures but the reliability of a task may depend on its type. The failure rate is unrelated: a probability of failure is associated to each couple (task type, machine). We consider different kind of mappings: in one-to-one mappings, each machine can process only a single task, while several tasks of the same type can be processed by the same machine in specialized mappings. Finally, general mappings have no constraints. The optimal one-to-one mapping can be found in polynomial time for particular problem instances, but the problem is NP-hard in most of the cases. For the most realistic case of specialized mappings, which turns out to be NP-hard, we design several polynomial time heuristics and a linear program allows us to find the optimal solution (in exponential time) for small problem instances. Experimental results show that the best heuristics obtain a good throughput, much better than the throughput achieved with a random mapping. Moreover, we obtain a throughput close to the optimal solution in the particular cases where the optimal throughput can be computed.
\end{abstract}

\section{INTRODUCTION}

In this paper, we study the problem of optimizing the throughput for micro-factories subject to failures. Microfactories are production systems composed of cells, each one performing a particular task on complex micro-components that pass through them. The probability for a fault to arise in these cells is high, so taking faults into account is mandatory when scheduling a production. In this context, faults are however not only attached to the processing unit, as it is commonly assumed for computer based distributed systems, but also to the tasks. In a production system a task may indeed be complex to perform, for instance due to some hard manipulation, with an impact on its success ratio. If the same robot is able to perform different tasks, it may generate less faults on simple tasks than on difficult ones.

To produce a micro-product, several tasks, each characterized by a task type, must be performed by the cells in an order fixed by a precedence graph. In the micro-factory, the robots that compose the cells must however be configured before being able to process a type of task. So the issue we face is to map several tasks of different types onto a set of cells, or machines, with the objective of optimizing the number of products that output the system, in spite of the faults.
In a first study [1], we have tackled the particular case in which faults only depend on the task type. In this paper we are interested in studying the impact of a fault model linked to both tasks and machines. Our specific use case is a micro-factory, more a production system than a distributed computing system, but the results presented in this paper are more generally applicable to distributed production systems or to distributed systems where the fault probability is attached not only to resources, but also to tasks.

The paper is organized as follows. The micro-factory context and related works are presented in Section II. Section III gives a more formal presentation of the micro-factories and of the failure model. Section IV presents the optimization problems tackled in the paper. The complexity study and results are given in Section V. In the rest of the paper, we focus on a particular variant of the problem, which is NP-hard: our aim is to find a specialized mapping which maximizes the throughput of a linear chain application. In Section VI, we provide several methods to solve this problem: (i) an integer linear programming formulation of the problem which allows us to find the optimal solution for small problem instances, and (ii) polynomial time heuristics for general instances. An extensive set of simulations is detailed in Section VII, and demonstrates the efficacy of our heuristics. Finally, we conclude in Section VIII.

\section{Context And STATE OF The ART}

Micro-factories are production units designed to produce pieces composed of micro-metric elements [2]. Today's microfactories are composed of micro-robots able to carry out basic operations through elementary actuators as piezo-electric beams (e.g., for gripping), stick-slip systems, etc. As these robots are usually teleoperated by a human operator, only simple tasks can be done. To perform more complex operations and to improve their efficiency, micro-factories need to be automated and robots need to be grouped in cells. Then cells will be put together and they will cooperate to produce complex assembled pieces, as it is done for macroscopic productions. Due to the piece, actuator and cell sizes, it is however impossible for human operators to directly interfere with the physical system. So it needs a highly automated command. The complexity of this command makes it mandatory to develop a distributed system to support this control. So, the 
cell group results in a distributed system that is very similar to a distributed computing platform. However, at this scale the physical constraints are not totally controlled so there is a need to take faults into account in the automated command.

The main issue for fault tolerant systems [3] is to overcome the failure of a node, a machine or a processor. To deal with those faulty machines, the most common method used in distributed systems is to replicate [4] the data. Those models assume that failures are attached to a machine. So the probability to get one product as a result is highly increased when the task is replicated on several machines. Once all the replicated jobs are done, a vote algorithm [5] is often used to decide which result is the right one. However, in our case the products are physical objects and therefore can not be replicated.

In real-time systems, another model called WindowConstrained [6] model can be used. In this model one considers that, for $y$ messages, only $x(x \leq y)$ of them will reach their destination. The $y$ value is called the Window. The looses are not considered as a failure but as a guarantee: for a given network a Window-Constrained Scheduling [7], [8] can guarantee that no more than $x$ messages will be lost for every $y$ sent messages. The Window-Constrained based failure model is adapted to a distributed system, the micro-factory. But in this paper, the objective function makes us use the failure model as the ratio $x / y$. In any case, the issue is to guarantee the output of a given number of products. Once an allocation of tasks to machines has been given, we can compute the number of products needed as input of the system and guarantee the output for the desired number of products.

\section{FRAMEWORK}

We outline in this section the characteristics of the applicative framework and target platform. Finally, we describe and motivate the failure model that we use in this work.

\section{A. Applicative framework}

We consider a set $\mathcal{N}$ of $n$ tasks: $\mathcal{N}=\left\{T_{1}, T_{2}, \ldots, T_{n}\right\}$. Each task $T_{i}(1 \leq i \leq n)$ is applied successively on a set of products. We wish to produce $x_{\text {out }}$ products as an output, and the total number of products being processed by a task may depend on the allocation: we process more than $x_{\text {out }}$ products since some losses may occur because of failures, as explained later in Section III-C. Note that all products are identical. When the context is not ambiguous, we may also design task $T_{i}$ by $i$ for clarity, as for instance in the figures.

A type is associated to each task as the same operation may be applied several times to the same product. Thus, we have a set $\mathcal{T}$ of $p$ task types with $n \geq p$ and a function $t:[1 . . n] \rightarrow \mathcal{T}$ which returns the type of a task: $t(i)$ is the type of task $T_{i}$, for $1 \leq i \leq n$.

The application is a directed acyclic graph (DAG) in which the vertices are tasks, and edges represent dependencies between tasks. An example of application with $n=5$ tasks is represented on Figure 1. In the top branch of the DAG, we need to finish the processing of task $T_{1}$ on one product before

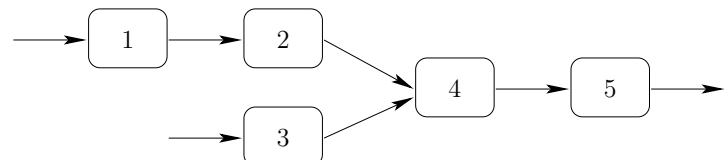

Figure 1: Example of application.

proceeding to task $T_{2}$. The join to task $T_{4}$ corresponds to the merge of two products, which produces a new unit of product composed of the two. Typically one instance of product from each predecessor in the graph is required to process with the joining task. Note that forks cannot be considered in this context as the output of one task is a physical component that cannot be split in two. Unlike data that can be easily replicated at every step of a DAG, an instance of a physical component is the result of all the preceding tasks and cannot be duplicated as it is material.

\section{B. Target platform}

The platform consists in a set $\mathcal{M}$ of $m$ machines: $\mathcal{M}=$ $\left\{M_{1}, M_{2}, \ldots, M_{m}\right\}$. All machines can be interconnected: the platform graph is a complete graph. A machine handles some of the tasks at a given speed: machine $M_{u}$ can perform the task $T_{i}$ onto one product in a time $w_{i, u}$. We also consider that tasks of the same type have the same execution time on a given machine, since they correspond to the same action to be performed on the products. Thus, we have:

$\forall i, i^{\prime} \in[1, n], \forall u \in[1, m], t(i)=t\left(i^{\prime}\right) \Rightarrow w_{i, u}=w_{i^{\prime}, u}$.

We neglect the communication time required to transfer a product from one machine to another. If a communication may not be negligible, we can always model it as a particular task with a dedicated machine (the machine responsible of the transfer of the product).

We are interested in producing the desired number of products rather than producing a particular instance of a product. So we consider that products are not identified: two products, on which the same sequence of tasks has been done, are exactly similar and we can use one or the other indifferently for further operations.

\section{Failure model}

An additional characteristic of our framework is that tasks are subject to failure. It may happen that a product is lost or damaged while a task is being executed on this product. For instance electrostatic strength may be accumulated on the actuator, and thus the piece will be pushed away rather than caught. Indeed, we work at a scale such that these electrostatic strengths are stronger than gravity.

Due to our application setting, we deal only with transient failures, as defined in [9]. The tasks are failing for some of the products, but we do not consider a permanent failure of the machine responsible of the task, as this would lead to a failure for all the remaining products to be processed and the unability to finish them.

One classical technique used to deal with failures is replication [4]. However, while replication is very useful for hardware failures of machines, we cannot use it in our framework since 
the products are not a data such as a numerical image that we need to process, but it is a physical object. The cost of these products is very low while the equipments are expensive. Thus, the only solution consists in processing more products than needed, so that at the end, the required number of finished products are brought out.

The failure rate of task $T_{i}$ performed onto machine $M_{u}$ is the percentage of failure for this task and is denoted $f_{i, u}=\frac{l_{i, u}}{b_{i, u}}$, where $l_{i, u}$ is the number of lost products each time $b_{i, u}$ products have been processed $\left(l_{i, u} \leq b_{i, u}\right)$.

\section{OPTIMIZATION PROBLEMS}

Now that the framework has been clarified, we formalize in this section the various optimization problems that we wish to solve. Our goal is to assign tasks to machines so as to optimize some key performance criteria. The solution to one problem is thus an allocation function $a:[1 . . n] \rightarrow[1 . . m]$ which returns for each task the machine on which it is executed. Thus, if $a(i)=u$, task $T_{i}$ is executed on machine $M_{u}$, and the processing of one product for this task takes a time $w_{i, u}$.

We first discuss the objective criteria that we want to optimize. Then we introduce the different rules of the game that can be used in the definition of the allocation function $a$. The complexity of these various problems is discussed in Section V.

\section{A. Objective function}

In our framework, several objective functions could be optimized. For instance, one may want to produce a mapping of the tasks on the machines as reliable as possible, i.e., minimize the total number of products to input in the system. Rather, we consider that products are cheap, and we focus on a performance criteria, the throughput. The goal is to maximize the number of products processed per time unit, making abstraction of the initialization and clean-up phases. This objective is important when a large number of products must be produced.

Rather than maximizing the throughput of the application, we rather deal with the period, which is the inverse of the throughput. First we introduce the fractional number $x_{i}$, which is the average number of products required to output one product out of the system for task $T_{i}$. We can compute $x_{i}$ recursively for any application. Let $T_{j}$ be the (unique) successor of $T_{i}$, if it exists (remember that we do not allow forks in the application graph). For tasks with no successor, we set $x_{j}=1$, which means that $T_{i}$ needs to output one product. Then, if task $T_{i}$ is assigned to machine $M_{u}$, we have

$$
x_{i}=\frac{1}{1-f_{i, u}} \times x_{j}=\frac{b_{i, u}}{b_{i, u}-l_{i, u}} \times x_{j},
$$

where the fraction represents the number of products needed per successful product. Starting from the nodes with no successor, we can then compute $x_{i}$ for each task $T_{i}$.

We are now ready to define the period of a machine: it is the time needed by a machine to execute all the tasks allocated onto this machine in order to produce one final product out of the system. Formally, we have

$$
\operatorname{period}\left(M_{u}\right)=\sum_{1 \leq i \leq n \mid a(i)=u} x_{i} w_{i, u} .
$$

The period of machine $M_{u}$ is the sum, for each task allocated to that machine, of the average number of products $\left(x_{i}\right)$ needed to output one product, multiplied by the speed $\left(w_{i, u}\right)$ of that task onto that machine. The slowest machine will slow down the whole application, thus we aim at minimizing the largest machine period. The machines realizing this maximum are called critical machines. If $M_{c}$ is a critical machine, then $\operatorname{period}=\operatorname{period}\left(M_{c}\right)=\max _{M_{u} \in \mathcal{M}} \operatorname{period}\left(M_{u}\right)$.

Note that minimizing the period is similar to maximizing the throughput.

\section{B. Rules of the game}

In this section, we classify several variants of the optimization problem that has been introduced. A task must always be processed by one unique machine (allocation function), but different rules can be enforced about what a machine can process.

1) One-to-one mappings: In this first class of problems, a machine can compute only one single task. This rule of the game is enforced with the following constraint, meaning that a machine cannot compute two different tasks:

$$
\forall 1 \leq i, i^{\prime} \leq n \quad i \neq i^{\prime} \Rightarrow a(i) \neq a\left(i^{\prime}\right) .
$$

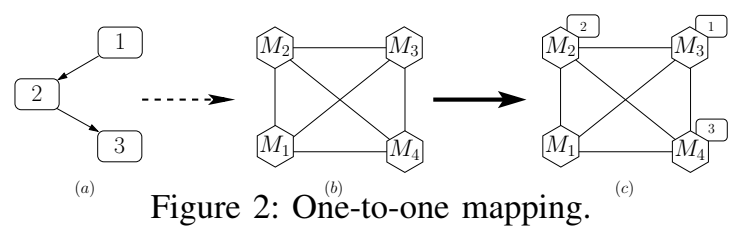

On Figure 2, we have an application graph $(a)$ that must be mapped on a platform graph $(b)$. The result is shown in $(c)$, where we can see that one machine can handle only one task. Thus this mapping is quite restrictive because we must have at least as many machines as tasks.

2) Specialized mappings: We have dedicated machines that can realize only one type of tasks. But task types are not dedicated to machines, so two machines may compute different tasks of the same type.

For instance, let us consider five tasks $T_{1}, T_{2}, T_{3}, T_{4}, T_{5}$ with the following types: $t(1)=t(3)=t(5)=1$ and $t(2)=t(4)=2$. If machine $M_{3}$ computes task $T_{1}$, it could also execute $T_{3}$ and $T_{5}$ but not $T_{2}$ and $T_{4}$. As types are not dedicated to machines, $T_{5}$ could also be assigned to another machine, for instance $M_{1}$. This situation is described on Figure 3.

The following constraint expresses the fact that a machine cannot compute two tasks of different types:

$$
\forall 1 \leq i, i^{\prime} \leq n \quad t(i) \neq t\left(i^{\prime}\right) \Rightarrow a(i) \neq a\left(i^{\prime}\right) .
$$




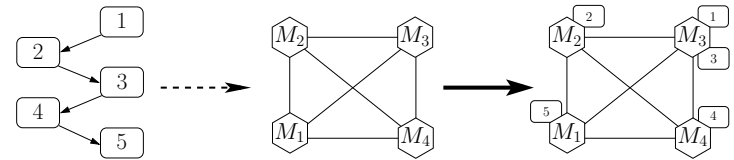

Figure 3 : $^{(a)}$ Specialized mapping (task types: $t\left(1^{(c)}\right)=t(3)=$ $t(5)=1$ and $t(2)=t(4)=2)$.

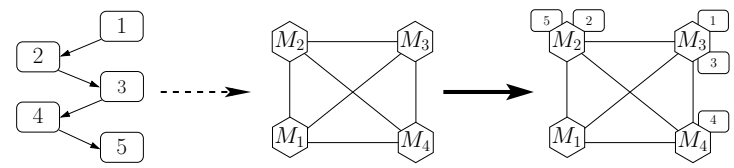

Figure $4:$ General mapping ${ }^{(b)}$ (task types: $t(1) \stackrel{(c)}{=} t(3)=1$, $t(2)=t(4)=2$ and $t(5)=3)$.

3) General mappings: A machine can compute any task regardless of its type, thus there are no constraints. An example of this case is shown on Figure 4.

\section{Complexity Results}

Complexity results are classified depending on the mapping rules. We start with one-to-one mappings, then we focus on specialized and general ones.

\section{A. Complexity of one-to-one mappings}

For one-to-one mappings, we can refine the problem complexity depending on the application class. We are particularly interested in linear chain applications, as in the example of Figure 1. Indeed, the problem remains polynomial for such applications (Theorem 1), while it turns out to be NP-hard for general applications (Theorem 2).

In this section, we introduce a new notation: $F_{i}=\frac{1}{1-f_{i, a(i)}}$.

Theorem 1. Given a linear chain application and a set of homogeneous machines $\left(w_{i, u}=w\right.$ for all $\left.i, u\right)$, finding the one-to-one mapping which maximizes the throughput can be done in polynomial time.

Proof: For a linear chain application with dependencies from task $T_{i}$ to task $T_{i+1}$, for $1 \leq i \leq n-1$, the average number of products $x_{i}$ needed to output one product out of the system and performed by the task $T_{i}$ can be computed thanks to the $F_{j}$, with $j \geq i$, see Section IV-A:

$$
x_{i}=F_{i} \times x_{i+1}=\prod_{i \leq j \leq n} F_{j} .
$$

Of course, the values of $F_{j}$ depend on the allocation function. Thus, the period $\operatorname{period}\left(M_{a(i)}\right)=\operatorname{period}(i)$ of the machine $M_{a(i)}$ on which the task $T_{i}$ is assigned to is $x_{i} \times w_{i, a(i)}=x_{i} \times w$. Since all $F_{j}$ values are greater than 1 , we have $x_{1}=\max _{1 \leq i \leq n} x_{i}$, and the period is constrained by the machine on which task $T_{1}$ is executed. The goal is thus to minimize the product $\prod_{1 \leq j \leq n} F_{j}$, in order to maximize the period.

Since the mapping is required to be one-to-one, we create a bipartite graph with one node per task $T_{j}(1 \leq j \leq n)$ on one side, one node per machine on the other side. The cost of an edge from task $T_{j}$ to machine $M_{u}$ is then set to $-\log \left(1-f_{j, u}\right)$, so as to transform the previous product into a sum.

Then, we can find in polynomial time a minimum weight matching in this bipartite graph, for instance using the Hungarian algorithm [10], [11]. This matching corresponds to an assignment of tasks to machines which minimizes $\prod_{1<j \leq n} F_{j}$, and thus it is equivalent to a one-to-one mapping which has a minimum period.

Note that this reasoning does not hold anymore with heterogeneous machines $\left(w_{i}, w_{u}\right.$ or $\left.w_{i, u}\right)$, since the bottleneck task is not necessarily $T_{1}$ in such cases. The complexity remains open for such cases.

However, if we consider general applications rather than restricting to linear chains, the problem becomes NP-hard.

Theorem 2. Finding the optimal one-to-one mapping is NPhard, even with constant processing costs $w$ and failure rates which depend on machines $\left(f_{i, u}=f_{u}\right.$ for $\left.1 \leq i \leq n\right)$.

Proof: We consider the following decision problem: given a period $K$, is there a one-to-one mapping whose period does not exceed $K$ ? The problem is obviously in NP: given a period and a mapping, it is easy to check in polynomial time whether it is valid or not.

The NP-completeness is obtained by reduction from 3PARTITION [12], which is NP-complete in the strong sense. Let $\mathcal{I}_{1}$ be an instance of 3-PARTITION: given a set $\left\{z_{1}, \ldots, z_{3 n}\right\}$ of $3 n$ integers, and an integer $Z$ such that $\sum_{1 \leq j \leq 3 n} z_{j}=n Z$, does there exist $n$ independent subsets $B_{1}, \cdots, B_{n}$ of $\left\{z_{1}, \cdots, z_{3 n}\right\}$ such that for all $1 \leq i \leq n$, $\sum_{z_{j} \in B_{i}} z_{j}=Z$ ?

We build the following instance $\mathcal{I}_{2}$ with $3 n+1$ tasks and processors:

- the application consists in $n$ linear chains of 4 tasks sharing the same final task $T^{(4)}$ : for $1 \leq i \leq n$, $T_{i}^{(1)} \rightarrow T_{i}^{(2)} \rightarrow T_{i}^{(3)} \rightarrow T^{(4)}$

- $w=1$ (constant processing cost);

- $f_{3 n+1}=0$ (machine $M_{3 n+1}$ never fails);

- for $1 \leq u \leq 3 n, f_{u}=\frac{2^{z u}-1}{2^{z} u}$

- $K=2^{Z}$.

Note that the size of $\mathcal{I}_{2}$ is polynomial in the size of $\mathcal{I}_{1}$. Indeed, since 3-PARTITION is NP-complete in the strong sense, we could encode $\mathcal{I}_{1}$ in unary, and thus the size of the instance would be in $O(n Z)$. Moreover, the values of $f_{u}$ can be encoded in binary and thus their size is polynomial in the size of $\mathcal{I}_{1}$.

Now we show that $\mathcal{I}_{1}$ has a solution if and only if $\mathcal{I}_{2}$ has a solution. Suppose that $\mathcal{I}_{1}$ has a solution. We construct the allocation function $a$ such that $a\left(T^{(4)}\right)=3 n+1$, i.e., the last task is processed by the reliable processor, and, for $1 \leq i \leq n$, tasks $T_{i}^{(1)}, T_{i}^{(2)}, T_{i}^{(3)}$ are allocated to the three processors such that $z_{u} \in B_{i}$. Since all $w$ are equal to 1 , the period of the mapping is constrained by one of the $T_{i}^{(1)}$ tasks, and their period is $P_{i}=\prod_{z_{u} \in B_{i}} \frac{1}{1-f_{u}}$. Taking the logarithm, $\log _{2}\left(P_{i}\right)=\sum_{z_{u} \in B_{i}} \log _{2}\left(\frac{1}{1-f_{u}}\right)=\sum_{z_{u} \in B_{i}} \log _{2}\left(2^{z_{u}}\right)=Z=$ 
$\log _{2}(K)$, that means $P_{i}=K$ for $1 \leq i \leq n$, and $\mathcal{I}_{2}$ has a solution.

Suppose now that $\mathcal{I}_{2}$ has a solution. The critical resource is still one of the $T_{i}^{(1)}$, since $w=1$. For each of these machines, we must have $\log _{2} P_{i} \leq Z$, and thus $\sum_{u \in \text { alloc }} z_{u}=Z$, where alloc $_{i}$ represents the set of indices of the four processors allocated to the $i^{t h}$ chain. To minimize this quantity, we can build a solution in which the reliable processor is processing task $T^{(4)}$, and then the problem amounts to 3-PARTITION the $z_{u}$. Therefore, $\mathcal{I}_{1}$ has a solution. This concludes the proof.

This NP-hardness result illustrates the additional difficulty of having a failure probability which depends both on tasks and machines. Indeed, the problem can be solved in polynomial time with fully heterogeneous machines $\left(w_{i, u}\right)$ when the failure rates are identical for all machines $\left(f_{i, u}=f_{i}\right.$ for each machine), because we are then able to compute $x_{i}$ for each task, independently of the mapping (see [1]).

\section{B. Complexity of specialized and general mappings}

In [1], we proved that the problem of finding the optimal specialized or general mapping is NP-hard, even for a linear chain application with constant processing costs $w$, and when failure probabilities are independent of the machines $\left(f_{i, u}=\right.$ $f_{i}$ ). Therefore, the problem remains NP-hard when considering more general values of failure probabilities. This illustrates the additional complexity of considering more general mapping rules rather than restricting to one-to-one mappings.

\section{SOlVING THE SPECIALIZED MAPPING PROBLEM}

In the practical setting of micro-factories, general mappings are not really useful because of the unaffordable reconfiguration costs. Indeed, if a machine is processing tasks of different types, one needs to reconfigure the machine between operations.

However, when the number $m$ of machines is greater than the number $p$ of task types, it is always possible to find a specialized mapping, since each machine is able to process all the tasks of a same type. The key point is then to find $m$ (or less) groups of tasks of the same type to be assigned to the $m$ machines of the platform. Even if we restrict to specialized mappings and linear chain applications, this problem is already NP-hard, as explained in Section V-B.

Thus, we present in the following a linear program and six heuristics that return a mapping, by grouping tasks of same type onto machines.

\section{A. Linear programming for the specialized mapping}

In this section, we present a linear program to solve the specialized mapping problem presented in Section IV-B2. This linear program is a Mixed Integer Program (MIP) because it uses both integer and rational variables. Solving a MIP is NPcomplete, however efficient solvers such as Cplex [13] makes it possible to solve small problem instances in a reasonable time. The following MIP implementation allows us to validate the relevance of the scalable heuristics that we present in the next section.

In the following, the two indices $i$ and $u$ denote respectively a task $T_{i}(1 \leq i \leq n)$ and a machine $M_{u}(1 \leq u \leq m)$.

The parameters of the linear program are the following:

- $w_{i, u}$ is the time needed by the task $T_{i}$ to perform one product onto the machine $M_{u}$;

- $f_{i, u}$ is the failure rate of task $T_{i}$ on machine $M_{u}$.

The variables needed to define the MIP are the following: - $x_{i}$ is the average number of products that the task $T_{i}$ has to perform to output one product out of the system;

- For any pair $\left(T_{i}, M_{u}\right)$ we denote $a_{i, u} \in\{0,1\}$ as the mapping of $T_{i}$ onto the machine $M_{u}: a_{i, u}=1$ if the task $T_{i}$ is performed by the machine $M_{u}$ and 0 otherwise;

- For any pair $\left(M_{u}, j\right)$ we denote $t_{u, j} \in\{0,1\}$ such as $t_{u, j}=1$ if the machine $M_{u}$ is specialized to perform tasks of type $j$ and 0 otherwise;

- $K \in \mathbb{Q}$ is a rational number which represents the upper bound on the period for all machines.

The objective function is to minimize the period $K$, but several constraints must be enforced to have a valid mapping function $(a)$, and a correct number of product $(x)$.

- We ensure that each task $T_{i}$ is performed by one and only one machine $M_{u}$ :

$$
\forall i \quad \sum_{u} a_{i, u}=1
$$

- We ensure that each machine $M_{u}$ is dedicated to at most one type $j$ :

$$
\forall u \quad \sum_{j} t_{u, j} \leq 1
$$

- We ensure that each task $T_{i}$ of type $j=t(i)$ can be performed only by one machine $M_{u}$ which is specialized upon the type $t(i)$. This constraint is not in contradiction with the fact that several tasks of same type $j$ can be performed by the machine $M_{u}$ :

$$
\forall u \forall i \quad a_{i, u} \leq t_{u, t(i)}
$$

- We ensure that the average number of products that the task $T_{i}$ has to perform depends on the mapping of $T_{i}$ but also on the number of products that the task $T_{i+1}$ has to perform to output one product out of the system.

$$
\forall u \forall i \quad x_{i} \geq \frac{1}{1-f_{i, u}} a_{i, u} \times x_{i+1}
$$

This formula can be transformed into the following linear equation:

$$
\forall i \forall u \quad x_{i} \geq \frac{1}{1-f_{i, u}} x_{i+1}-\left(1-a_{i, u}\right) M A X_{x_{i}}
$$

where $M A X_{x_{i}}$ is an upper bound of $x_{i}$ such that $x_{i} \leq$ $M A X_{x_{i}}=\prod_{i \leq j \leq n} \frac{1}{1-\max _{1 \leq u \leq m}\left(f_{j, u}\right)}$. 
- The period of each machine $M_{u}$ depends on the mapping and its value is bounded by $K$ :

$$
\forall u \quad \sum_{i} a_{i, u} \times x_{i} w_{i, u} \leq K
$$

This non-linear formula can be transformed into the following linear inequations. In order to make the linearization possible, we define a new positive rational variable $y_{i, u}=a_{i, u} \times x_{i}$ for every task $T_{i}$ and for every machine $M_{u}$. So the previous equation can be rewritten into the equation (7) under the constraints (8):

$$
\begin{gathered}
\forall u \quad \sum_{i} y_{i, u} w_{i, u} \leq K \\
\begin{cases}\forall i \forall u & y_{i, u} \leq a_{i, u} M A X_{x_{i}} \\
\forall i \forall u & y_{i, u} \leq x_{i} \\
\forall i \forall u & y_{i, u} \geq x_{i}-\left(1-a_{i, u}\right) M A X_{x_{i}}\end{cases}
\end{gathered}
$$

The objective is to minimize the period under the previous constraints, thus we get the following MIP:

$$
\left\{\begin{array}{l}
\text { Minimize } K \\
\text { under the constraints (3), (4), (6), (7), (8) }
\end{array}\right.
$$

\section{B. Heuristics}

Since faults occur depending on the task and the machine, we are not able to compute the number of products the task $T_{i}$ has to perform before knowing which task is assigned to which machine. The six heuristics presented here are executed by starting with the last task of the application graph and going backward to the first one.

H1: Random heuristic. A task $T_{i}$ is assigned to a machine $M_{u}$ if $M_{u}$ is free or if $M_{u}$ is already specialized to tasks of the type $t(i)$. If none of these conditions are fulfilled, we try the next machine $M_{u+1}$ and so on until an available machine is found.

H2: Binary search heuristic 1. This heuristic aims at optimizing the potential of the machines, i.e., the goal is to assign to each machine a set of tasks for which it is efficient. Thus, we start by sorting, for each machine $M_{u}$, the set of $w_{i, u}$, for $1 \leq i \leq n$, in ascending order. Then, $\operatorname{rank}_{i, u}$ represents the rank of $T_{i}$ in the ordered set for $M_{u}$.

The heuristic performs a binary search on the period between 0 (best case) and the time required to perform sequentially all the tasks on a machine (worst case). For each value of the search, all tasks are assigned greedily (from $T_{1}$ to $T_{n}$ ) to machines.

We try to assign the task $T_{i}$ to a machine such that $\operatorname{rank}_{i, u}$ is minimum. If the rank equals one, this means that the potential of $M_{u}$ for this task is optimal. In case of equality (several machines of identical rank for $T_{i}$ ), machines are sorted by non-decreasing values of $w_{i, u}$. Of course, the assignment can be done only if the machine was not already specialized to a type which is different from $t(i)$, and if the fixed period is not exceeded. Otherwise we try to assign $T_{i}$ to the next machine, according to their priority order for this task. If no machine is able to process $T_{i}$, then no assignment is found and we try a larger period. If all tasks can be correctly assigned, we try a smaller period.

H3: Binary search heuristic 2. This heuristic is the same as $\mathrm{H} 2$ except that, for the assignment, the machines are sorted by their heterogeneity level in descending order. The idea is to preserve homogeneous machines for the last tasks. The heterogeneity level of $M_{u}$ is computed as the standard deviation of its $w_{i, u}$ values. Each task is assigned to the most heterogeneous machine capable of handling it. Note that for this heuristic, slow machines may be used instead of powerful ones, because of their heterogeneity level.

H4: Best performance heuristic. This heuristic assign a task $T_{i}$ to the machine $M_{u}$ with the best performance value for that task. The performance value of $M_{u}$ for $T_{i}$ is computed by $w_{i, u} \times f_{i, u} \times x_{i}$.

H4w: Faster machine heuristic. This heuristic is the same that $\mathrm{H} 4$ except that the faster machine is selected $\left(w_{i, u} \times x_{i}\right)$ without taking into account the failure rate in the assignment process.

H4f: Reliable machine heuristic. This heuristic is the same that $\mathrm{H} 4$ except that the most reliable machine is selected $\left(f_{i, u} \times x_{i}\right)$ without taking into account the speed in the assignment process.

\section{EXPERIMENTS}

In this section, we compare the six heuristics that give scheduling solutions to the specialized mapping problem with $w_{i, u}$ and $f_{i, u}$ for linear chain applications. The results are computed by a simulator, developped in $\mathrm{C}++$. The performance of each heuristic is measured by its period in $\mathrm{ms}$.

Recall that $m$ is the number of machines, $p$ the number of types, and $n$ the number of tasks. Each point in the figures is an average value of 30 simulations where the $w_{i, u}$ are randomly chosen between 100 and $1000 \mathrm{~ms}$, for $1 \leq i \leq n$ and $1 \leq u \leq$ $m$. Similarly, failure rates $f_{i, u}$ are randomly chosen between $0.5 \%$ and $2 \%$ (i.e., $1 / 200$ and $1 / 50$ ), unless stated otherwise.

\section{A. Specialized mappings with $m$ and $p$ fixed}

In this first set of experiments, the number of machines $m$ and the number of task types $p$ are fixed, and we plot the period for each heuristic as a function of the number of tasks $n$.

Figure 5 shows that $\mathrm{H} 1$ and $\mathrm{H} 4 \mathrm{f}$ are not very competitive. Indeed, minimizing the failure rate does not prevent from choosing a slow machine and so getting a long period. For the next experiment, only the other heuristics are plotted. In Figure 6, H4 is slightly under the others. That is explained by the $f_{i, u}$ factor used by H4. Two major factors are in competition here, the speed and the reliability. A large platform is set (100 machines) to see the difference between those two factors. In Figure 7, H4w shows up to be better than the others. As a conclusion of this first set of experiments, we can say that the machine speed seems to be a more important criteria than its reliability when taking assignment choices.

To study more precisely the effect of failure rates, platforms with a high failure rate (up to $10 \%$ ) are used. Figure 8 shows that periods are increasing dramatically with the number of tasks. In that special case only $\mathrm{H} 2$ is performing well. 


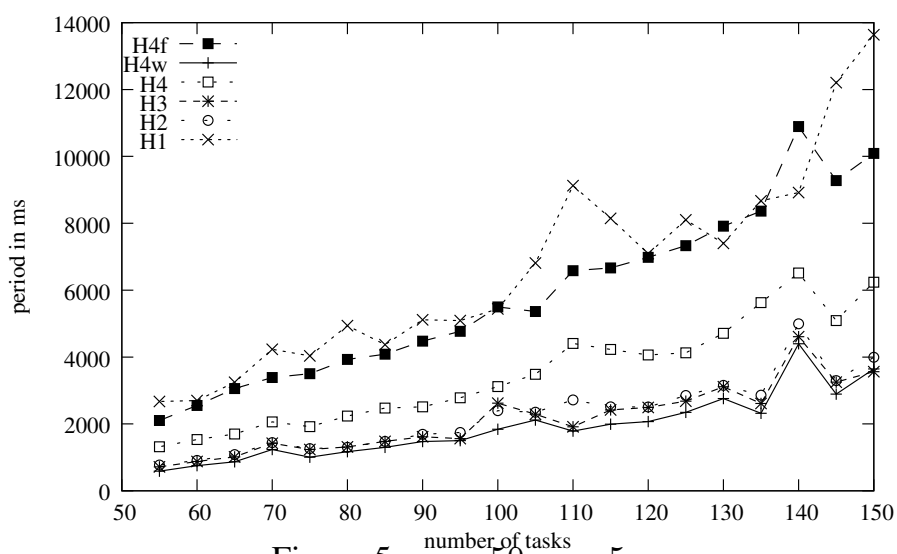

Figure 5: $m=50, p=5$.

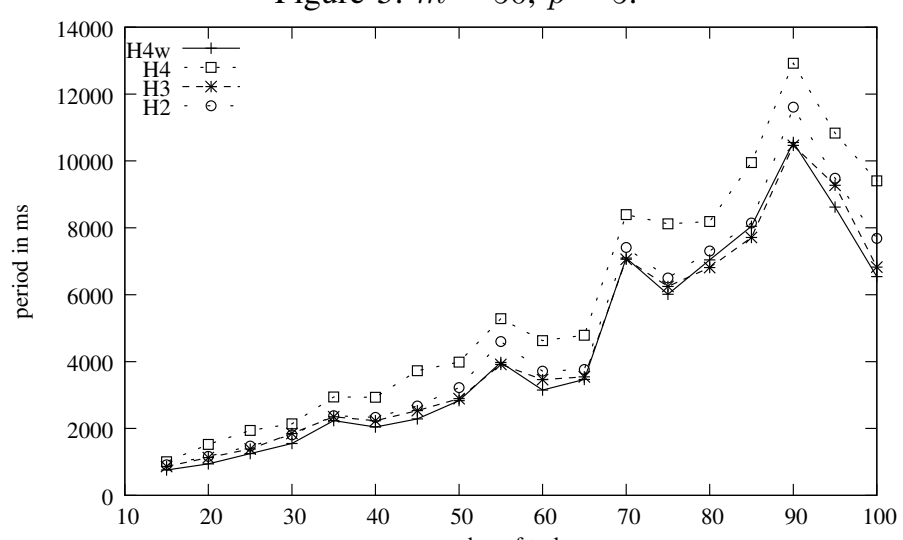

Figure 6: $m=10, p=2$.

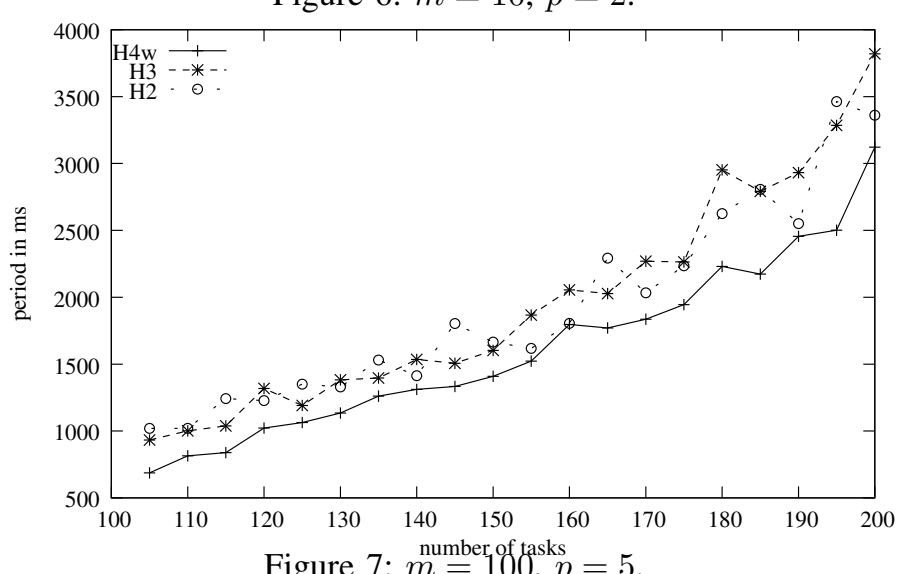

Figure 7: $m=100, p=5$.

\section{B. One-to-one mappings with $m$ and $n$ fixed}

As shown in section V-A, considering the one-to-one mapping, an optimal solution can be found in polynomial time only if the failure is attached only to tasks $\left(f_{i, u}=f_{i}\right.$ for $1 \leq u \leq m)$. Thus, a platform with 100 machines, 100 tasks and failures defined by $f_{i}$ is set. We plot the period as a function of the number of types $p$ and run 100 simulations for each dot of the figure. Figure 9 shows H2, $\mathrm{H} 3, \mathrm{H} 4 \mathrm{w}$ and the optimal one-to-one solution (OtO). For a better visibility the other heuristics are ignored here. H4w has the best performance and is very close to the optimal when the number of types is low. We can also see that when the

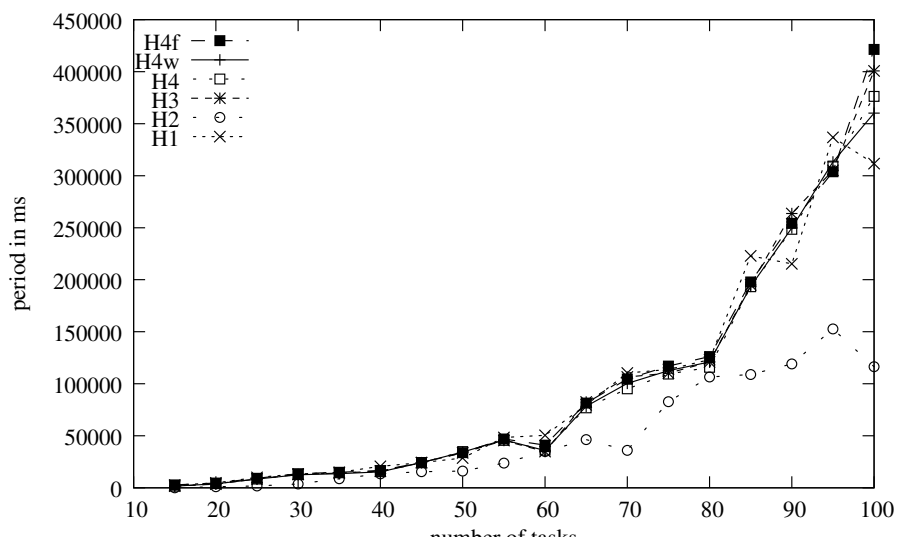

Figure 8: $m=10, \stackrel{\text { number of tasks }}{=} f_{i, u} \leq 0.1$.

number of types is high, all heuristics tend to have the same performance. This is explained by the fact that with $p$ close to $m$, the way of creating the groups of tasks is less crucial. Results are very encouraging and show that $\mathrm{H} 2, \mathrm{H} 3$ and $\mathrm{H} 4 \mathrm{w}$ are respectively at a factor of $1.84,1.75$ and 1.28 from the optimal solution.

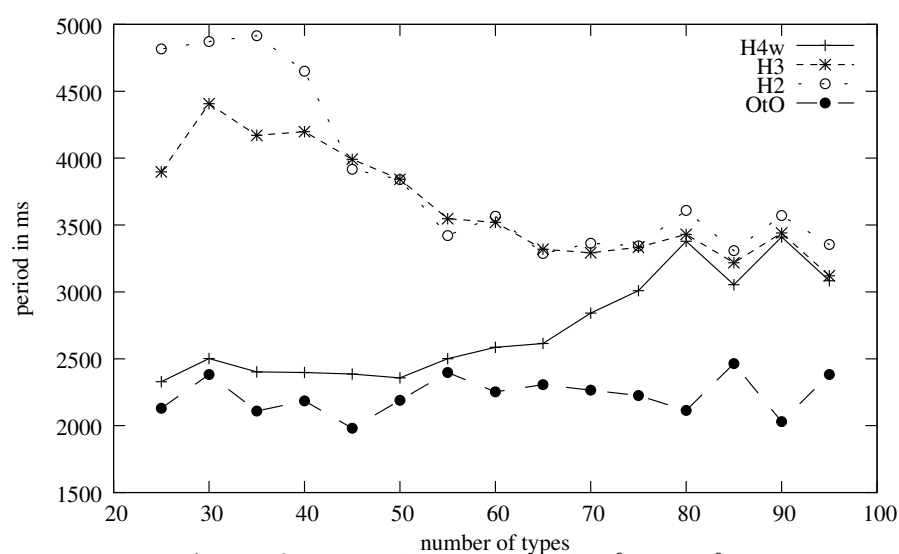

Figure 9: $m=100, n=100, f_{i, u}=f_{i}$.

\section{Comparison with the linear program}

This last set of experiments compares our heuristics to the mixed integer linear program (MIP) described in Section VI-A. We restrict the study to small problem instances, so that we are able to derive a solution for the linear program, and results are reported only if 30 successful experiments over 60 trials are obtained with the MIP. Those "MIP-compatible" platforms are selected and the heuristics are run on them.

In the first experiment, we use a platform with 5 machines, and the application has 4 types. We are then able to target applications with up to 15 tasks. Figure 10 shows that $\mathrm{H} 4 \mathrm{w}$ is once again the best heuristic but $\mathrm{H} 2$ and $\mathrm{H} 4$ are close. To measure that difference, Figure 11 shows the normalization of the heuristics with the MIP solution. Results reveal that $\mathrm{H} 2$, $\mathrm{H} 3$ and $\mathrm{H} 4 \mathrm{w}$ are respectively at a factor of $1.73,1.58$ and 1.33 from the optimal.

For the next experiment, we use a platform with 9 machines, and the application has between 5 and 20 tasks of 4 different 
types. For visibility reason, we discard results of $\mathrm{H} 1$ and $\mathrm{H} 4 \mathrm{f}$ from the figure. Figure 12 shows that with more than 15 tasks, the MIP is not able to find solutions anymore.
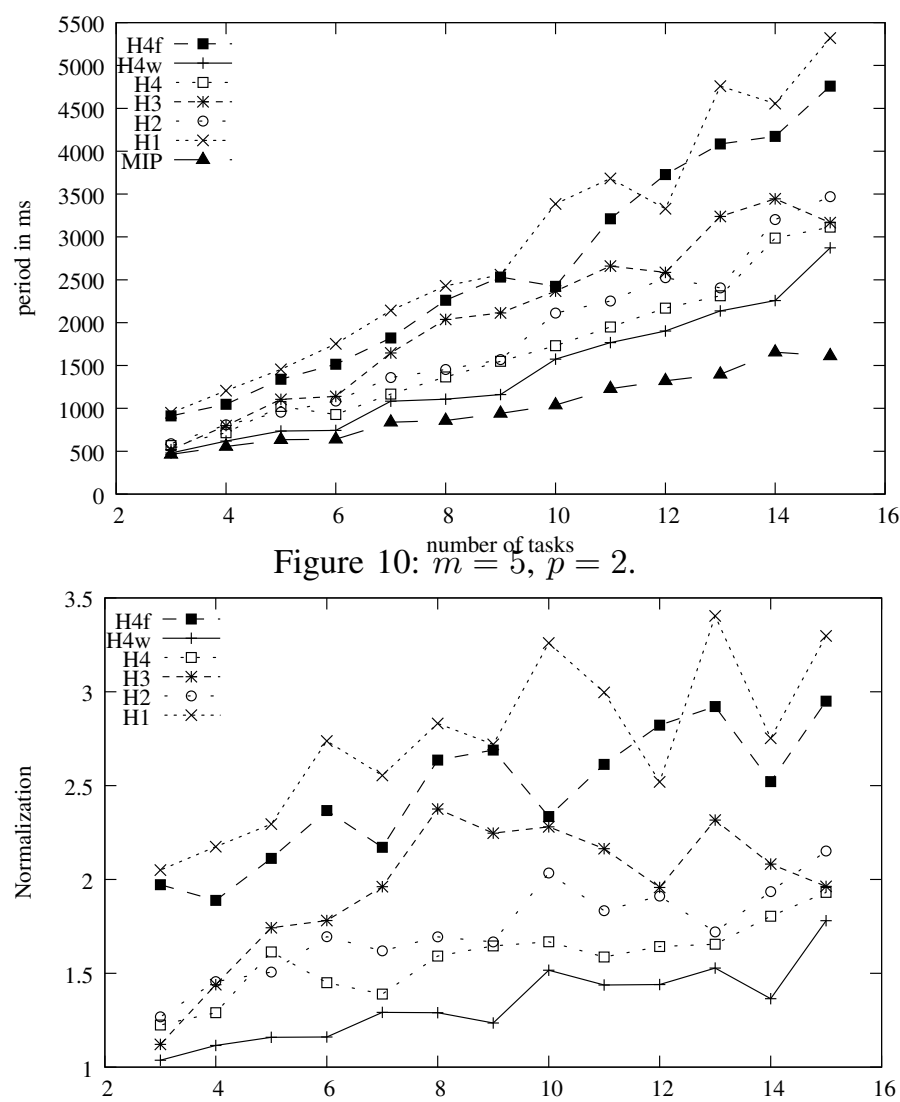

Figure 11: $m=5, p=2$. Normalization with the MIP.

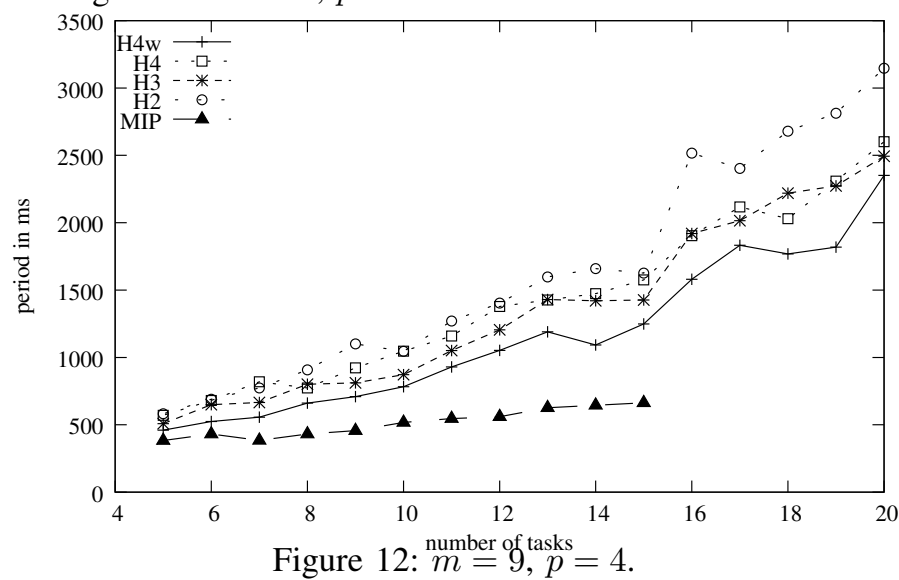

\section{CONCLUSION}

In this paper, we investigate a throughput optimization problem in the context of micro-factories subject to failures. The problem consists in assigning tasks of tree-shaped application graphs to machines. The failures that occur in the system depend on both the task and the machine on which the task is assigned. We proved that the problem to find an optimal oneto-one mapping for linear chain onto homogeneous machines is polynomial while the problem becomes NP-hard for in-tree one-to-one mappings or for specialized and general mappings. Since general mappings are not usable in practice because of reconfiguration costs, we focused on specialized mappings and proposed several polynomial heuristics to solve the problem when the graph is a linear chain. Also, a mixed linear programming formulation of the problem is given to allow us to evaluate our heuristics by comparing experimental results to the optimal, considering small problem instances. These experimental results showed that the most performing solution is obtained by $\mathrm{H} 4 \mathrm{w}$. This heuristic focuses on the execution speed and does not take into account the failure rate. The comparison between $\mathrm{H} 4 \mathrm{w}$ and the optimal solutions that can be found respectively for one-to-one mappings and specialized mappings (onto small platforms thanks to the linear program) showed that $\mathrm{H} 4 \mathrm{w}$ is respectively at a factor of 1.28 and 1.33 from the optimal. This is a very promising result, but somehow expected, which means that if we produce fast enough we overcome the faults.

As future work, an interesting problem would be to consider that the instances of a same task can be computed by several machines. Thus, the workload of a task would be divided and the throughput could be improved.

\section{REFERENCES}

[1] A. Benoit, A. Dobrila, J.-M. Nicod, and L. Philippe, "Throughput optimization for micro-factories subject to failures," in Proc. of the 8th International Symposium on Parallel and Distributed Computing, Jul. 2009, available as Research Report RR-LIP-2009-02 at graal.ens-lyon. $\mathrm{fr} / \sim$ abenoit/papers/RR-LIP-2009-02.pdf.

[2] M. Tanaka, "Development of desktop machining microfactory," Journal RIKEN Rev, vol. 34, pp. 46-49, April 2001, iSSN:0919-3405.

[3] V. P. Nelson, "Fault-tolerant computing: Fundamental concepts," Computer, vol. 23, no. 7, pp. 19-25, 1990 .

[4] W. Cirne, F. Brasileiro, D. Paranhos, L. F. W. Góes, and W. Voorsluys, "On the efficacy, efficiency and emergent behavior of task replication in large distributed systems," Parallel Computing, vol. 33, no. 3, pp. 213-234, 2007.

[5] B. Parhami, "Voting algorithms," IEEE Transactions on Reliability, vol. 43, no. 4, pp. 617-629, Dec 1994

[6] R. West and C. Poellabauer, "Analysis of a window-constrained scheduler for real-time and best-effort packet streams," in Proc. of the 21st IEEE Real-Time Systems Symp. IEEE, 2000, pp. 239-248.

[7] R. West, Y. Zhang, K. Schwan, and C. Poellabauer, "Dynamic windowconstrained scheduling of real-time streams in media servers," 2004. [Online]. Available: citeseer.ist.psu.edu/article/west03dynamic.html

[8] R. West and K. Schwan, "Dynamic window-constrained scheduling for multimedia applications," in ICMCS, Vol. 2, 1999, pp. 87-91. [Online]. Available: citeseer.ist.psu.edu/west98dynamic.html

[9] P. Jalote, Fault Tolerance in Distributed Systems. Prentice Hall, 1994.

[10] H. W. Kuhn, "The hungarian method for the assignment problem," Naval Research Logistics Quarterly, vol. 2, pp. 83-97, 1955.

[11] I. S. Duff and J. Koster, "On algorithms for permuting large entries to the diagonal of a sparse matrix," SIAM Journal on Matrix Analysis and Applications, vol. 22, pp. 973-996, 2001.

[12] M. R. Garey and D. S. Johnson, Computers and Intractability, a Guide to the Theory of NP-Completeness. W.H. Freeman and Company, 1979.

[13] "ILOG CPLEX: High-performance software for mathematical programming and optimization," http://www.ilog.com/products/cplex/. 